\title{
Politique
}

\section{Le pouvoir prédominant dans les statuts des partis : le cas du} NPD

\section{Vincent Lemieux}

Numéro 14, automne 1988

Sport et politique et Le NPD

URI : https://id.erudit.org/iderudit/040601ar

DOI : https://doi.org/10.7202/040601ar

Aller au sommaire du numéro

\section{Éditeur(s)}

Société québécoise de science politique

\section{ISSN}

0711-608X (imprimé)

1918-6584 (numérique)

Découvrir la revue

\section{Citer cet article}

Lemieux, V. (1988). Le pouvoir prédominant dans les statuts des partis : le cas du NPD. Politique, (14), 63-82. https://doi.org/10.7202/040601ar d'utilisation que vous pouvez consulter en ligne.

https://apropos.erudit.org/fr/usagers/politique-dutilisation/ 


\title{
LE POUVOIR PRÉDOMINANT DANS LES STATUTS DES PARTIS: LE CAS DU NPD
}

\author{
Vincent Lemieux \\ Université Laval
}

Dans son ouvrage sur l'analyse politique contemporaine Robert Dahl distingue quatre méthodes qui peuvent être utilisées pour l'étude de l'influence, celle-ci étant définie par lui comme «un rapport entre des acteurs par lequel l'un d'entre eux amène les autres à agir autrement qu'ils ne l'auraient fait sans cela» ${ }^{1}$.

Premièrement, le chercheur, dit Dahl, peut présumer que l'influence d'un acteur est en relation étroite avec sa position dans une hiérarchie officielle ou semi-officielle.

Deuxièmement, le chercheur peut faire confiance à des juges bien placés, à qui il demande d'évaluer l'influence des acteurs.

Troisièmement, l'enquêteur peut essayer de percer la façade que constituent la position formelle et la réputation, en étudiant la participation à la prise de décision.

Quatrièmement, le chercheur peut aller plus loin sur certe voie en mesurant l'importance des activités reliées à la prise de décision: par exemple, prendre l'initiative, fixer des priorités, obtenir l'assentiment, légitimer, exécuter.

La méthode que nous voulons proposer pour l'étude du pouvoir dans les statuts des partis politiques consiste pour une

1. L'analyse politique contemporaine, Paris, Laffont, 1973, p. 53.

Politique, 14 (Automne 1988). 
bonne part dans la combinaison de la première et de la quatrième méthode de Dahl. Elle porte sur la hiérarchie officielle des positions occupées par les acteurs, mais elle tient aussi compte des activités reliées à ces positions. Elle tient compte également de la représentativité de ceux qui occupent les positions dans la hiérarchie officielle, ce qui est un élément nouveau par rapport à la première et à la quatrième méthode de Dahl.

La méthode vise à établir le lieu où réside l'autorité organisatrice la plus extensive, au sens de la capacité et de la représentativité à la fois. C'est en ce lieu que se trouve, selon nous, le pouvoir officiel prédominant dans un parti. Cet approche cherche à dépasser les limites de l'analyse traditionnelle du pouvoir dans les partis, pour proposer un ensemble de concepts opératoires qui puissent être utilisés aussi bien pour l'étude du pouvoir officiel que pour celle du pouvoir effectif.

\section{Les limites de l'analyse traditionnelle}

Il n'existe pas à notre connaissance d'analyse systématique de la distribution du pouvoir dans les statuts des partis politiques. Les quelques études existantes sont ou bien normatives, ou bien purement descriptives. Par contre, beaucoup d'auteurs, à la suite d'Ostrogorski et de Michels ${ }^{2}$, ont conduit des analyses du pouvoir effectif exercé dans les partis, qu'il soit conforme ou non au pouvoir officiel, prévu dans les statuts (quand ils existent).

L'ouvrage classique de Duverger ${ }^{3}$ demeure le plus souvent cité dans ce domaine. L'auteur prétend que «les partis actuels se

2. M. OSTROGORSKI dans La démocratie et les partis politiques, Paris, CalmannLévy, 1903, parlait de «formalisme», alors que c'est à R. MICHELS, Les partis politiques, Paris, Flammarion, 1911, qu'on doit l'expression de la loi d'airain de l'oligarchie. Cette thèse a été contestée entre autres par $\mathrm{S}$. J. ELDERSVELD dans son livre sur les partis américains (Political Parties A Bebavioral Analysis, Chicago, Rand NcNally, 1964).

3. M. DUVERGER, Les partis politiques, Paris, Colin, 1951. 
définissent beaucoup moins par leur programme ou la classe de leurs adhérents que par la nature de leur organisation» (pp. IX$\mathrm{X}$ ) et consacre toute la première partie de son livre à la structure des partis. La question du pouvoir y est omniprésente, en particulier dans le troisième chapitre, consacré à la direction des partis. La façon dont elle est traitée comporte cependant trois limites importantes.

Premièrement, la notion de pouvoir demeure floue et ambiguë. Elle réfere parfois au pouvoir comme possession de moyens («les titulaires du pouvoir») et parfois au pouvoir comme exercice d'une action ( la personnalisation du pouvoir»). C'est là une confusion courante dans l'étude du pouvoir.

Deuxièmement, Duverger s'intéresse beaucoup plus au pouvoir de désignation qu'au pouvoir d'instigation. ${ }^{4}$ Il explore les différentes modalités par lesquelles les désignations aux différents postes de commande des partis sont plus centralisées en fait qu'elles devraient l'être en droit, mais il prête beaucoup moins d'attention au pouvoir par lequel les dirigeants et les autres élites, une fois en place, influencent les autres membres du parti, ou sont influencés par eux. Les différents enjeux du pouvoir d'instigation ne sont pas distingués les uns des autres.

Troisièmement, le rapport entre les pouvoirs de désignation et les pouvoirs d'instigation n'est pas traité systématiquement, même si l'ouvrage de Duverger contient en cette matière comme en d'autres des aperçus éclairants. En particulier, la base de représentation, qui est importante pour l'exercice du pouvoir d'instigation, n'est traitée que de façon implicite.

Notre proposition de recherche vise à dépasser ces limites et à élaborer une méthode qui permette de cerner de façon rigoureuse le lieu où réside le pouvoir prédominant dans un parti.

4. Nous empruntons ce terme à B. De JOUVENEL dans De la politique pure, Paris, Calmann-Lévy, 1963. 


\section{Une proposition de recherche}

Les statuts d'une organisation, comme les lois ${ }^{5}$ ou règlements, sont faits d'un ensemble de règles qui définissent des positions occupées par des acteurs et des relations entre ces positions. Nous considérons les relations comme des relations de pouvoir quand les règles accordent à des acteurs, en certaines positions, le droit de contrôler des décisions qui concernent leurs moyens d'action ou ceux d'autres acteurs en d'autres positions.

Nous employons le terme de position dans un sens très général pour désigner les différents postes, instances, catégories de membres etc, qui sont identifiés dans les statuts d'un parti.

Le terme de décision est employé lui aussi dans un sens très général. C'est toute action qui suppose un choix entre au moins deux options: voter ou ne pas voter, choisir tel candidat plutôt que tel autre, etc.

Les moyens d'action, qui sont les enjeux du pouvoir, peuvent être distingués en deux grandes catégories: les ressources matérielles, humaines ou informationnelles qui sont transmises d'une position à l'autre; et les attributions qui ont trait à l'utilisation des ressources, aux statuts et aux rôles des acteurs, ou aux normes de l'action. Le pouvoir est allocatif quand il a pour enjeu des ressources, et il est organisateur quand il a pour enjeu des attributions.

Le pouvoir organisateur est un pouvoir plus déterminant que le pouvoir allocatif puisqu'en agissant sur les attributions on agit sur les ressources, alors que l'inverse est moins vrai. Par exemple, si on enlève le droit de vote à un membre, on lui enlève la possibilité de transmettre une information par son vote, alors que si on le prive d'informations avant de voter on ne le prive pas pour autant de son vote.

5. Sur l'étude du pouvoir dans les lois, voir V. LEMIEUX «L'analyse structurale des lois", Revue Canadienne de Science Politique, XV, 1, mars 1982: 67-84. 
Nous considérons que le pouvoir prédominant est de l'ordre de l'organisation plutôt que de l'allocation, et que ceux qui disposent du pouvoir prédominant exercent plus de pouvoir qu'ils en subissent.

Nous avons choisi les statuts du Nouveau Parti Démocratique (NPD) du Canada ${ }^{6}$ pour une première application de notre méthode, qui consiste à identifier le lieu du pouvoir officiel prédominant dans un parti. Cette identification sera faite en trois étapes.

$\left.1^{\circ}\right)$ Après avoir fait un relevé des différentes positions définies dans les statuts, le pouvoir officiel ou l'autorité ${ }^{7}$ de ces positions sera recensée, en distinguant l'autorité allocative (sur les ressources) de l'autorité organisatrice (sur les attributions) car nous faisons le postulat que le pouvoir officiel prédominant en est une d'autorité organisatrice.

$2^{\circ}$ ) La composition des positions qui ont de l'autorité organisatrice est ensuite étudiée. Il est postulé que les acteurs qui ont le plus de capacité sont ceux qu'on retrouve toujours inclus dans les positions qui ont de l'autorité organisatrice. Il est évident, pour prendre des cas extrêmes, que dans un congrès ou dans un conseil, le leader ou le président du parti a plus de capacité, étant donné sa participation à différentes instances du parti, qu'un simple délégué ou encore qu'un représentant des jeunes du parti.

$3^{\circ}$ ) À capacité égale entre deux positions, il est postulé que celle des deux qui a la base de représentation, ou la représentativite ${ }^{8}$ la plus grande, a aussi le plus de pouvoir officiel. Le postulat

6. Nous utilisons l'édition 1986 des statuts, telle que modifiée au congrès fédéral de 1985. Pour le moment nous ne tenons pas compte de l'annexe, portant sur les règles de procédure.

7. Dans la suite nous utilisons de façon commode les termes de pouvoir officiel et d'autorité comme synonymes, même si le terme d'autorité a un sens qui ne recouvre pas parfaitement celui de pouvoir officiel.

8. La notion de représentativité est employée ici et dans la suite de façon neutre et dans un sens strictement quantitatif, sans égard à la qualité de la représentation. 
repose sur deux constats qui sont faits couramment. D'abord plus la base de représentation est grande plus la légitimité l'est également, du moins dans les systèmes démocratiques, ce qui assure aux acteurs les plus représentatifs un plus grand potentiel dans l'exercice du pouvoir. Ensuite, plus la base est étendue, plus un représentant a de liberté par rapport à cette base, qui est moins susceptible de le contrôler constamment que si la base est restreinte. Les acteurs les plus représentatifs seraient pour cela moins susceptibles de subir du pouvoir que les acteurs qui sont moins représentatifs.

Notre proposition de recherche est donc la suivante: le pouvoir officiel prédominant appartient aux acteurs qui ont le plus de représentativité parmi ceux qui ont la plus grande capacité en autorité organisatrice.

\section{Les positions identifiées dans les statuts}

Ou trouvera la liste des positions en annexe, dans l'ordre de leur apparition aux différents articles des statuts du NPD. Cette liste comprend plus de soixante positions. La variété est donc grande, dans des staruts qui sont relativement courts (environ 2500 mots). Plusieurs raisons expliquent cette grande variété. Notons entre autres:

- le souci de la démocratie, qui se manifeste par un système de représentation relativement compliqué;

- l'existence de membres affiliés, qu'on ne trouve pas en d'autres partis;

- la place faite aux jeunes, qui entraîne la définition de plusieurs positions qui les concernent;

- la place faite aux femmes et aux francophones, qui se traduit par des positions «associées» à d'autres positions ou par la création de positions spécifiques; 
- la nécessité de prévoir des relations avec les sections provinciales du parti et avec l'aile parlementaire, à la Chambre des communes.

\section{L'autorité allocative}

Si on en tient pas compte des relations d'inclusion, d'intersection et d'exclusion qui concernent la composition des positions, les statuts du NPD contiennent plus de cent relations de pouvoir ayant pour enjeu des attributions ou des ressources. Il n'est pas question de les reproduire ici ${ }^{9}$. Après quelques remarques sur les pouvoirs qui ont pour objet des ressources, nous nous concentrerons sur le pouvoir organisateur, celui qui concerne les attributions, dont nous avons posé qu'il était plus déterminant que le pouvoir qui porte sur les ressources.

Nous reviendrons, dans l'étude de la représentativité, sur le pouvoir concernant des ressources humaines où les acteurs d'une position désignent le ou les membres qui occuperont une autre position. Les pouvoirs sur les ressources matérielles se trouvent à peu près tous dans l'article IV, qui a pour titre: cotisations, redevances et partage des revenus. Les principales positions impliquées sont le Conseil fédéral, les sections provinciales du parti et le Parti fédéral, ainsi que les membres individuels et les membres

9. On trouvera un exemple concret du repérage d'un ensemble de relations de pouvoir dans "L'analyse structurale des lois". Limitons-nous ici au premier paragraphe des statuts du NPD où sont énoncées des relations de pouvoir. Il se lit comme suit (article II, paragraphe 2): «Le Président est l'interprète des présents statuts. Toute interprétation de sa part peut être renversée par un vote majoritaire de tous les membres présents et votant à une réunion de l'Exécutif, du Conseil ou du congrès". Le paragraphe prévoit d'abord que le Président a le droit d'allouer de l'information, en interprétant les statuts. Il y a là une première relation de pouvoir, de nature allocative. La suite de l'article indique que l'Exécutif, le Conseil ou le congrès sont les positions qui sont contrôlées par le Président dans cette allocation. Les trois mêmes positions peuvent cependant, dans une autre relation de pouvoir, contrôler les attributions du Président en renversant son interprétation par un vote majoritaire. La première relation de pouvoir (celle du Président sur l'Exécutif, sur le Conseil ou sur le congrès) peut donc être annulée par la deuxième. 
affiliés. Le Conseil fédéral apparaît comme prédominant en ces matières, en ce qu'il établit la formule de partage des droits d'adhésion et des cotisations entre toutes les sections provinciales et le Parti fédéral. C'est aussi le Conseil fédéral qui gère le traitement du Secrétaire et du Secrétaire associé, les principaux fonctionnaires du Parti (article VII).

Les pouvoirs sur les ressources informationnelles sont plus divers. Ils concernent les préavis et les projets de résolution ayant rapport avec un congrès (article $\mathrm{V}$ ), les données sur le nombre de membres en règle (article VII et IX), les rapports que l'Exécurif doit faire au Conseil (article IX), les avertissements que le Secrétaire doit transmettre à des candidats, à des associations de circonscription et à des partis provinciaux (article XIII). Comme c'est généralement le cas en matière de pouvoir sur l'information, les positions impliquées sont diverses et aucune ne prédomine de façon nette sur les autres.

\section{L'autorité organisatrice}

Les pouvoirs organisateurs sur les attributions sont ceux qui nous intéressent le plus, étant donné notre proposition de recherche. Quatre enjeux principaux du pouvoir organisateur se dégagent des statuts du NPD: la modification des statuts, la convocation d'un congrès extraordinaire, les questions de reconnaissance et de discipline touchant différentes positions dans le Parti, et la possibilité de faire des déclarations qui engagent le Parti.

$\left.1^{\circ}\right)$ Le dernier article, l'article XIV, pose que les statuts peuvent être modifiés par un vote de deux tiers de tous les délégués présents et votants à tout congrès. C'est le pouvoir organisateur le plus général qui soit.

$2^{\circ}$ L'article $V$ prévoit qu'il puisse y avoir congrès extraordinaire. Le Conseil peut le convoquer si et quand il le juge nécessaire. Il doit le convoquer si une majorité des partis provinciaux 
ou si une majorité des associations de circonscription fédérale le demande. Il s'agit ici d'une autorité organisatrice plus procédurale que substantielle, mais notons quand même que le Conseil, les partis provinciaux et les associations de circonscription fédérale détiennent de l'autorité en ces matières, le Conseil ayant l'avantage d'être un déclencheur de congrès extraordinaire moins lourd et plus facile à mobiliser que les deux autres. Ajoutons que tous les membres du Conseil sont délégués à un congrès, ce qui n'est pas le cas de tous les membres des partis provinciaux et de tous les membres des associations de circonscription fédérale.

$3^{\circ}$ ) D'autres dispositions concernent les problèmes de reconnaissance de certaines positions, ou encore de discipline des membres. Les sections provinciales du parti et les jeunes néo-démocrates sont les principaux enjeux de ce pouvoir organisateur. Des partis provinciaux, il est dit à l'article XI qu'ils sont autonomes, mais que leurs statuts et principes ne doivent pas entrer en conflit avec ceux du Parti fédéral. S'il y a conflit, c'est le Conseil fédéral du Parti qui est investi du pouvoir de décider si une organisation continue ou non d'être un parti provincial, sous réserve d'un appel au congrès.

Il est dit également des jeunes néo-démocrates qu'ils sont constitués en section autonome, mais que leurs statuts n'entreront pas en conflit avec ceux du Parti fédéral (article $\mathrm{X}$ ). Ce sont les partis provinciaux qui accordent une charte à une section provinciale des jeunes, mais s'il n'y a pas de section provinciale, le Conseil fédéral peut accorder des chartes à des clubs de jeunes.

L'autorité du Conseil fédéral est aussi affirmée dans les questions de discipline. C'est aux partis provinciaux de voir à la discipline de leurs membres individuels et de certains membres affiliés, mais le Conseil fédéral est chargé de voir à la discipline d'autres membres affiliés (article XII). C'est lui également qui a 
le pouvoir d'intervenir dans les mises en candidature, si le parti provincial impliqué n'a pas pris les mesures qui s'imposent (article XIII).

$4^{\circ}$ Nous considérons le pouvoir de faire des déclarations au nom du Parti comme un pouvoir organisateur, qui porte sur les attributions de celui-ci, dans la mesure où ces déclarations définissent des normes qui s'imposent au Parti et qui doivent guider son action. Deux instances ont ce pouvoir, le Conseil fédéral et l'Exécutif, mais l'autonomie du Conseil est plus grande que celle de l'Exécutif. Celui-ci «a le pouvoir d'émettre des déclarations au nom du Parti sous réserve d'en faire rapport au Conseil et d'obtenir l'approbation du Conseil» (article IX), alors que le Conseil «a pleins pouvoirs d'émettre au nom du Parti des déclarations politiques et des déclarations électorales qui s'accordent avec les décisions des congrès du Parti fédéral, de formuler des déclarations qui s'accordent avec la philosophie du Parti à l'égard des questions n'ayant pas encore été délibérées par le congrès» (article VIII).

On voit que plusieurs positions du Parti ont de l'autorité organisatrice. La capacité en autorité organisatrice de ces positions est cependant plus ou moins étendue.

\section{La capacité en autorité organisatrice}

Tout compte fait, le Conseil fédéral apparaît comme l'instance avec l'autorité organisatrice la plus grande. Les membres sont délégués ex-officio au congrès du Parti, le Conseil peut convoquer un congrès extraordinaire, c'est lui qui est chargé de reconnaître ou non les principales sections du Parti (partis provinciaux et sections des jeunes) et de veiller à la discipline, et enfin son pouvoir de déclaration est supérieur à celui de l'Exécutif, qui est inclus en son sein. 
Pour établir la capacité respective des différentes positions incluses dans celle de Conseil fédéral, faisons en d'abord la liste. Ce sont (article VIII):

a) les dirigeants (élus par le congrès);

b) vingt autres membres élus par le congrès, dont dix doivent être des femmes;

c) deux membres élus par les députés fédéraux;

d) le leader, le président, le secrétaire et le trésorier de chaque parti provincial;

e) trois membres additionnels de chacune des sections provinciales (élus par le congrès provincial);

f) un membre représentant chacune des douze organisations affiliées qui regroupent le plus grand nombre de membres affiliés;

g) un membre jeune néo-démocrate de chacune des sections de jeunes néo-démocrates à charte provinciale (élu par le congrès provincial compétent);

h) des membres additionnels nommés par le parti provincial intéressé, quand il y a occupation de certains postes par la même personne.

De plus, le Conseil, avec l'approbation des deux tiers des membres présents et votants, peut coopter cinq membres additionnels.

Nous avons proposé de mesurer la capacité en autorité organisatrice d'une position donnée par l'autorité organisatrice des autres positions où cette position donnée est incluse. Il s'agit donc de voir dans quelles autres positions sont inclus les membres du Conseil et de noter l'autorité organisatrice de ces autres positions. On peut distinguer à cet égard quatre catégories de membres du Conseil, en notant que les membres du Conseil sont tous délégués au congrès fédéral. 
$\left.1^{\circ}\right)$ Certains des membres du Conseil ne sont inclus, en droit, que dans une vaste position d'autorité organisatrice, le congrès fédéral ou les congrès provinciaux, même si, en fait, ils peuvent être inclus en d'autres positions d'autorité. Ce sont les vingt autres membres élus par le congrès fédéral et les membres additionnels de chacune des sections provinciales.

$2^{\circ}$ ) D'autres membres sont aussi dépourvus, ou presque, d'autorité organisatrice de par leurs inclusions en dehors du Conseil. Ce sont les cinq membres additionnels cooptés par le Conseil, dont on ne signale pas qu'ils peuvent avoir d'autres inclusions, et aussi les représentants des organisations affiliées, auxquelles les statuts du Parti ne reconnaissent pas de pouvoir organisateur. On peut mettre dans la même catégorie les représentants des jeunes néo-démocrates, dans la mesure où leurs sections provinciales n'ont pas d'autorité organisatrice autre qu'envers elles-mêmes.

$3^{\circ}$ ) Les deux membres élus par les députés fédéraux ainsi que les leaders, présidents, secrétaires et trésoriers des partis provinciaux sont dans une situation un peu meilleure. De par leur participation aux instances des partis provinciaux (ce que les statuts du Parti fédéral ne couvrent pas) ils ont une autorité organisatrice importante envers ceux-ci. On a $\mathrm{vu}$, de plus, qu'une majorité de partis provinciaux ou une majorité des associations de circonscription fédérale peut convoquer, par l'intermédiaire du Conseil, un congrès extraordinaire. Sachant la place qu'occupent les dirigeants dans les partis provinciaux et celle qu'occupent les députés ou les aspirants-députés dans les associations de circonscription fédérale, on peut estimer que leur autorité organisatrice est supérieure à celle des deux premières catégories de membres du Conseil.

$\left.4^{\circ}\right)$ Ce sont toutefois ceux qu'on nomme les «dirigeants» du Parti qui ont, en tant que tels, la plus grande capacité en 
autorité organisatrice. Cela tient surtout à leur inclusion dans l'Exécutif du Parti. Quatorze autres membres du Conseil, choisis par lui, sont aussi membres de l'Exécutif. Ils ont ainsi la même capacité que celle des dirigeants, en particulier pour ce qui est du pouvoir, cependant limité, de faire des déclarations qui engagent le parti.

\section{La représentativité}

C'est donc le Conseil fédéral du NPD et en particulier les membres de l'Exécutif qui ont, d'après les statuts, le plus de capacité en autorité organisatrice. Qu'en est-il de leur représentativité?

Il y a deux catégories de membres à cet égard. Les dirigeants qui sont élus par le congrès, et d'autres membres de l'Exécutif, qui sont élus par le Conseil. Les dirigeants ont donc une base de représentation plus grande que celle des autres membres de l'Exécutif.

Les dirigeants comprennent (article VII, paragraphe 1):

a) un leader;

b) un président et un président associé, avec possibilité d'un président associé additionnel si les deux premiers sont du même sexe;

c) huit vice-présidents, dont quatre doivent être des femmes;

d) un trésorier.

Abstraction faite des quatorze membres élus par le Conseil, l'Exécutif comprend aussi le Président sortant de charge et le Président associé sortant de charge. Peuvent s'y ajouter un Président émérite et un Président honoraire, nommés par le congrès.

Le Conseil nomme aussi, en plus des quatorze membres qu'il élit, un Secrétaire et un Secrétaire associé, l'un desquels est de langue française et l'autre de langue anglaise. 


\section{Le lieu du pouvoir officiel prédominant}

D'après notre proposition de recherche, le pouvoir officiel prédominant dans le NPD serait donc concentré entre les mains des membres de l'Exécutif, élus ou nommés par le congrès, soir ceux qu'on nomme "les dirigeants» dans les statuts du parti.

Cette conclusion doit cependant être modulée par quelques considérations additionnelles, qui permettent de préciser le pouvoir de certains des membres de l'Exécutif.

Premièrement, la base officielle de représentation n'est pas faite uniquement de ce qui est prévu dans les statuts du Parti, mais aussi de la base électorale que peuvent avoir les membres sur le plan fédéral ou sur le plan provincial. Quand le leader ou un autre membre de l'Exécutif est un député fédéral, il dispose évidemment d'un avantage important sur les dirigeants du Parti qui ne sont pas des élus fédéraux ou provinciaux. Notons à cet égard qu'un député fédéral ne peut occuper le poste de président ni celui de président associé, même s'il est toléré qu'un président ou un président associé qui est élu député durant son mandat demeure en poste jusqu'à la fin de ce mandat.

Deuxièmement, la base de représentation des membres de l'Exécutif qui ne sont pas des dirigeants fait une différence entre eux. Elle peut même leur conférer une représentativité plus grande que celle de certains dirigeants. Par exemple un membre (non dirigeant) de l'Exécutif qui est un député provincial ou fédéral ou encore le président d'un parti provincial a sans doute un pouvoir de représentation plus grand qu'un vice-président qui est un simple délégué d'une section provinciale ou d'une organisation affiliée.

Troisièmement, des différences apparaissent dans la capacité en autorité organisatrice des dirigeants ou plus largement des membres de l'Exécutif, quand on tient compte d'autres documents officiels que les statuts. Pour s'en tenir uniquement aux règles 
de procédure qui sont annexées aux statuts, il est bien évident qu'elles conferent au Président d'assemblée (qui est généralement le Président, le Président associé ou un vice-président), lors des congrès, un pouvoir sur les attributions des délégués qui n'est pas négligeable. Il en est de même de la présidence du Conseil et de la présidence de l'Exécutif.

\section{Conclusion}

Nous avons voulu, dans cet article, définir et appliquer une voie rigoureuse de recherche qui permette d'établir, à partir des règles officielles d'une organisation, quelles sont les positions qui disposent du pouvoir officiel prédominant dans cette organisation. Le repérage de ces postes se fait en trois étapes. On identifie d'abord les positions qui ont de l'autorité organisatrice, celle qui porte sur les attributions des acteurs. Ensuite, on repère les acteurs qu'on retrouve toujours dans les positions d'autorité organisatrice, et on estime que leur capacité est plus grande que celle des autres. Enfin les acteurs dont l'autorité organisatrice a la plus grande capacité sont distingués entre eux selon leur représentativité. Ceux qui ont la base de représentation la plus étendue ont le plus de représentativité.

Appliquée aux statuts du NPD, cette méthode montre que plusieurs positions ont de l'autorité organisatrice, mais qu'on retrouve toujours les membres du Conseil et en particulier ceux de l'Exécutif dans ces positions d'autorité organisatrice, et que dans l'Exécutif ceux qu'on nomme les dirigeants ont plus de représentativité que les autres. Ce sont eux qui prédominent parce qu'ils sont les plus représentatifs parmi ceux qui ont le plus de capacité en autorité organisatrice.

Les résultats auxquels nous arrivons ne sont pas révolutionnaires. Ceux qui ont l'expérience des partis politiques savent bien que même si le congrès est l'instance suprême d'un parti et que 
le parti se présente sous un jour démocratique, certaines positions de par leur situation stratégique dominent les autres, malgré ce que peut laisser croire une lecture superficielle des statuts. Notre proposition de recherche et la méthode suivie pour la tester ont au moins le mérite de rendre explicites les fondements du pouvoir prédominant et la démarche à suivre pour l'établir.

Ajoutons que l'étude d'un seul cas ne permet pas de rendre tout à fait justice à la méthode. Les avantages ressortiraient mieux dans des études comparatives faites sur un même parti, dans des versions différentes de ses statuts, ou faites sur des partis différents entre eux. Ces études comparatives permettraient de montrer que certains partis ont des positions prédominantes qui sont plus concentrées que d'autres, ou encore que les modifications apportées aux statuts d'un parti vont dans le sens de la concentration ou de la déconcentration du pouvoir prédominant.

Rappelons enfin que la méthode a porté sur le pouvoir officiel ou l'autorité, alors que la science politique cherche plutôt à établir le pouvoir effectif des acteurs sociaux. Nous avons cependant la prétention de croire que certains des concepts et des propositions qui ont été utilisés dans cet article permettraient de faire progresser la recherche sur le pouvoir effectif, qui est plutôt stagnante depuis une vingtaine d'années.

Premièrement, la définition du pouvoir que nous avons proposée est opérationnalisable dans la recherche empirique. Il s'agit d'identifier des décisions, des acteurs qui participent à ces décisions, les moyens d'action (ressources ou attributions) qui sont l'enjeu des décisions et quels sont les acteurs que l'affectation des enjeux favorise ou défavorise. Il y a là une vue interactive et évolutive du pouvoir qui en vaut bien d'autres.

Deuxièmement, la distinction entre le pouvoir allocatif et le pouvoir organisateur (qu'il y aurait lieu de raffiner) nous semble nécessaire pour éviter une vue "aplatie» du pouvoir, de nature trop strictement behavioriste. Il existe des niveaux de pouvoir, 
qui s'impliquent les uns les autres, et qu'il est nécessaire de distinguer dans la recherche sur la distribution du pouvoir.

Troisièmement, l'article $a$ aussi introduit la notion de capacité en pouvoir organisateur. Les acteurs qui se retrouvent toujours dans les positions qui disposent de pouvoir organisateur auraient un avantage sur ceux qui ne s'y retrouvent pas toujours. Cette proposition semble évidente, mais comme bien d'autres elle a fait l'objet de peu de recherches empiriques.

Quatrièmement, notre proposition concernant la représentativité apparaît plus discutable, même si elle correspond assez bien à une des thèses de MICHELS ${ }^{10}$. Les représentants qui ont la base de représentation la plus grande seraient doublement favorisés, en ce qu'ils exerceraient le pouvoir avec plus de légitimité et qu'ils subiraient moins les contrôles de leur base, selon une certaine logique de l'action collective décortiquée, entre autres, par OLSON ${ }^{11}$. Là encore, il y a des pistes de recherche à explorer qui pourraient être instructives sur l'exercice du pouvoir.

Nous ajouterions, en terminant, que la recherche sur le pouvoir ne pourra vraiment progresser que si on utilise les mêmes concepts et les mêmes propositions, et ultimement la même théorie, pour l'étude du pouvoir officiel et pour celle du pouvoir effectif, ainsi d'ailleurs que pour celle du pouvoir idéal, tel qu'il est exprimé dans les idéologies et les utopies.

10. Pour cet auteur, en effet, les chefs ont d'autant plus d'autorité que leur base de représentation s'accroît.

11. M. OLSON, La logique de l'action collective, Paris, Presses Universitaires de France, 1978. 


\section{ANNEXE}

Voici la liste des positions, dans l'ordre de leur apparition aux différents articles des statuts du NPD:

Président (art. II)

Exécutif (art. II)

Conseil (art. II)

Congrès (art. II)

Membres individuels (art. III)

Membres affiliés (art. III)

Parti provincial du NPD (art. III)

Syndicats ouvriers et agricoles (art. III)

Coopératives (art. III)

Organisations féminines (art. III)

Autres groupes et organisations (art. III)

Organisation internationale, nationale, provinciale ou régionale (art. III)

Section provinciale ou régionale d'une organisation (art. III)

Section, loge ou division d'une section (art. III)

Groupe local ou organisation locale (art. III)

Membre d'une organisation affiliée (art. IV)

Congrès extraordinaire (art. V)

Association de circonscription fédérale (art. 5)

Secrétaire (art. V)

Section provinciale des jeunes du NPD (art. 5)

Club (de jeunes) à charte fédérale (art. 5)

Comité des résolutions du Congrès (art. V)

Délégué à un congrès du parti (art. V)

Membre individuel en règle (art. VI)

Membre affilié en règle (art. VI)

Organisation centrale constituée d'organisations affiliées (art. VI)

Caucus du parti au Parlement fédéral (art. VI) 
Jeune néo-démocrate (art. VI)

Délégués jeunes néo-démocrates (art. VI)

Jeune néo-démocrate (art. VI)

Section provincial des jeunes (art. VI)

Club des jeunes à charte fédérale (art. VI)

Exécutif provincial (art. VI)

Dirigeant (art. VII)

Leader (art. VII)

Président associé (art. VII)

Président associé additionnel (art. VII)

Vice-président (art. VII)

Trésorier (art. VII)

Président sortant de charge (art. VII)

Président associé sortant de charge (art. VII)

Leader intérimaire (art. VII)

Secrétaire associé (art. VII)

Président émérite (art. VII)

Président honoraire (art. VII)

Leader d'une section provinciale (art. VIII)

Président d'une section provinciale (art. VIII)

Secrétaire d'une section provinciale (art. VIII)

Trésorier d'une section provinciale (art. VIII)

Congrès provincial (art. VIII)

Comité de participation des femmes (art. VIII)

Comités permanents et spéciaux (art. VIII)

Commission bipartite sur les problèmes constitutionnels (art. VIII)

NPD - Québec (art. VIII)

Leader du NPD - Québec (art. VIII)

Services du NPD (art. VIII)

Section autonome des jeunes du NPD (art. X)

Jeune membre en règle (art. $\mathrm{X}$ )

Parti provincial en règle (art. $\mathrm{XI}$ )

Membres individuels d'une section provinciale (art. XII) 
Membres affiliés d'une section provinciale (art. XII)

Candidat (art. XIII)

Représentants mandatés d'une association de circonscription (art. VIII) 